\title{
Diffuse infiltrating retinoblastoma invading subarachnoid space
}

This article was published in the following Dove Press journal:

Clinical Ophthalmology

22 June 2011

Number of times this article has been viewed

\author{
Satoru Kase' \\ Kazuhiko Yoshida' \\ Shigenobu Suzuki ${ }^{2}$ \\ Koh-ichi Ohshima ${ }^{3}$ \\ Shigeaki Ohno ${ }^{4}$ \\ Susumu Ishida' \\ 'Department of Ophthalmology, \\ Hokkaido University Graduate School \\ of Medicine, Sapporo; ${ }^{2}$ Department \\ of Ophthalmic Oncology, National \\ Cancer Center Hospital, Tokyo; \\ ${ }^{3}$ Section of Ophthalmology, \\ Okayama Medical Center, Okayama; \\ ${ }^{4}$ Department of Ocular Inflammation \\ and Immunology, Hokkaido University \\ Graduate School of Medicine, \\ Sapporo, Japan
}

Correspondence: Kazuhiko Yoshida Department of Ophthalmology, Hokkaido University Graduate School of Medicine, Nishi 7, Kita I5, Kita-ku, Sapporo 060-8638, Japan

Tel +8II I706 5944

Fax +81117065948

Email kyoshida@med.hokudai.ac.jp
Abstract: We report herein an unusual case of diffuse infiltrating retinoblastoma involving the brain, which caused a patient's death 27 months after enucleation. An eight-year-old boy complained of blurred vision in his right eye (OD) in October 2006. Funduscopic examination showed optic disc swelling, dense whitish vitreous opacity, and an orange-colored subretinal elevated lesion adjacent to the optic disc. Fluorescein angiography revealed hyperfluorescence in the peripapillary region at an early-phase OD. Because the size of the subretinal lesion and vitreous opacity gradually increased, he was referred to us. His visual acuity was 20/1000 OD on June 20, 2007. Slit-lamp biomicroscopy showed a dense anterior vitreous opacity. Ophthalmoscopically, the subretinal orange-colored area spread out until reaching the mid peripheral region. A B-mode sonogram and computed tomography showed a thick homogeneous lesion without calcification. Gadolinium-enhanced magnetic resonance imaging showed a markedly enhanced appearance of the underlying posterior retina. Enucleation of the right eye was performed nine months after the initial presentation. Histopathology demonstrated retinal detachment and a huge choroidal mass invading the optic nerve head. The tumor was consistent with diffuse infiltrating retinoblastoma. The patient died due to brain involvement 27 months after enucleation. Ophthalmologists should be aware that diffuse infiltrating retinoblastoma may show an unfavorable course if its diagnosis is delayed.

Keywords: diffuse infiltrating retinoblastoma, subarachnoid space, optic nerve

\section{Introduction}

Diffuse infiltrating retinoblastoma, a rare atypical retinoblastoma, is characterized by a diffuse infiltration of tumor cells in the retina without any focal mass. Almost all patients with diffuse infiltrating retinoblastoma show unilateral sporadic retinoblastomas. The prognosis is better than for infantile retinoblastomas, if enucleation is conducted before metastasis. ${ }^{1,2}$ Herein, we report an unusual case of diffuse infiltrating retinoblastoma involving the brain, which caused the patient's death 27 months after enucleation.

\section{Case report}

An eight-year-old boy complained of blurred vision in his right eye (OD) in October 2006. His visual acuity was 20/63 OD. The left eye was normal. Funduscopic examination showed optic disc swelling, dense whitish vitreous opacity, and an orange-colored subretinal elevated lesion (Figure 1A, arrowhead) adjacent to the optic disc. Fluorescein angiography revealed hyperfluorescence in the peripapillary region at an early phase OD (Figure 1B). Although a diagnosis of atypical retinoblastoma was suspected, it was 

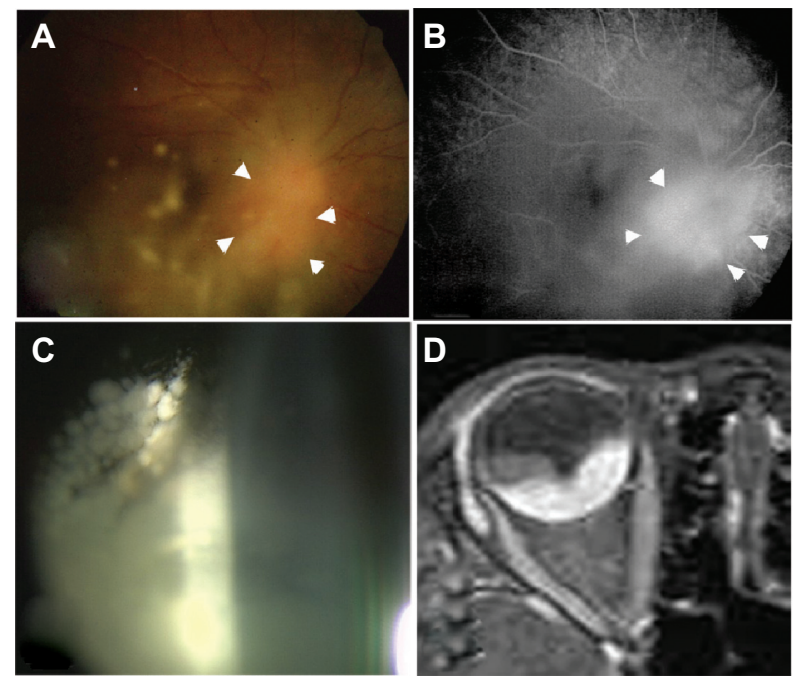

Figure I Fundus photograph (A), fluorescein angiography (B), slit-lamp biomicroscopy (C), and gadolinium-enhanced TI-weighted MRI of the right eye (D). A) The fundus was blurred due to the dense vitreous opacity. The optic disc was swollen, and there was an elevated orange-colored subretinal lesion situated adjacent to the optic disc (arrowhead). B) Hyperfluorescence in the initial phase (arrowhead) in the orange-colored subretinal lesion and optic disc. C) Whitish dense anterior vitreous opacity. D) A markedly enhanced appearance underlying the posterior retina.

difficult to differentiate from granulomatous uveitis at this stage. Because the size of the subretinal lesion and vitreous opacity gradually increased, he was referred to us.

His visual acuity was 20/1000 OD on June 20, 2007. Slit-lamp biomicroscopy showed a dense anterior vitreous opacity (Figure 1C). Ophthalmoscopically, the subretinal orange-colored area spread out until reaching the mid peripheral region. A B-mode sonogram and computed tomography showed a thick homogeneous lesion without calcification. Gadolinium-enhanced magnetic resonance imaging showed a markedly enhanced appearance of the underlying posterior retina (Figure 1D). Because atypical retinoblastoma was suspected, enucleation of the right eye was performed on July 3, 2007.

Histopathology demonstrated retinal detachment and a huge choroidal mass invading the optic nerve head (Figure 2A). In the retina, tumor cells invaded the ganglion cell layer without forming masses (Figure 2B). In the choroidal mass, tumor cells were tightly packed, and contained large hyperchromatic nuclei and scant cytoplasm. The tumor cells were consistent with retinoblastoma cells. Rosette formation was frequently observed (Figure 2C), but neither a necrotic area nor calcified lesion was found. There were infiltrating tumor cells in the parenchyma of the optic nerve and subarachnoid space (Figure 2D, arrow). The infiltration of tumor cells extended $10 \mathrm{~mm}$ into the subarachnoid space of the optic nerve up to the line of surgical transection.

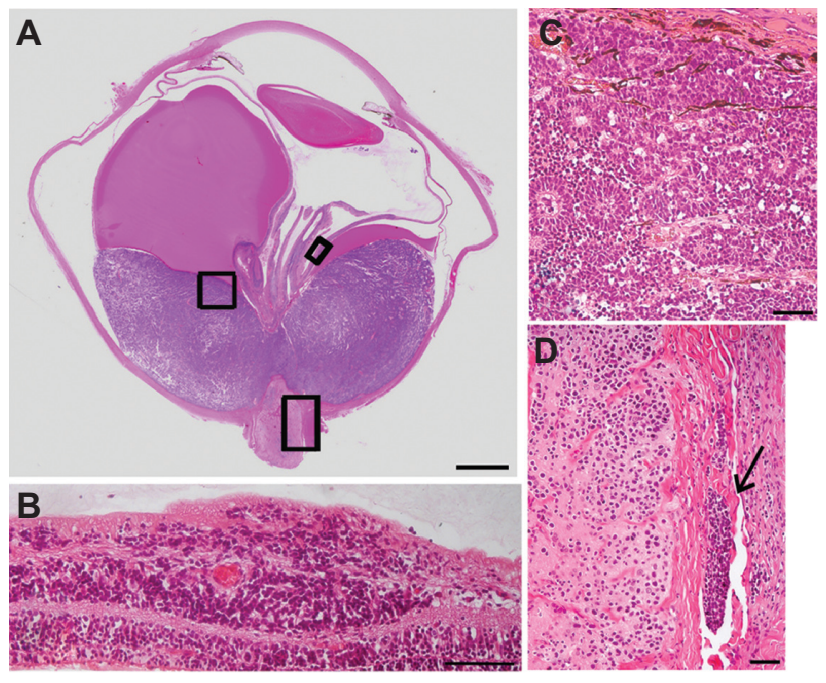

Figure 2 Histopathology of the enucleated eye. Hematoxylin and eosin staining. A) Retinal detachment with a huge choroidal mass invading the optic nerve head. Bar indicates $3 \mathrm{~mm}$. B) Tumor cells invaded the ganglion cell layer without forming masses. Bar indicates 50 um. C) Tumor cells were tightly packed, and contained large hyperchromatic nuclei and scant cytoplasm. Rosette formation was observed in the choroidal mass. Bar indicates 50 um. D) Tumor cells infiltrated the parenchyma of the optic nerve and subarachnoid space (arrow). Bar indicates 50 um.

Tumor cells were also observed in the choroidal veins, and along the path of the long posterior ciliary artery in the scleral canal. In contrast, there were no tumor cells in the anterior segments, including the corneal endothelium and anterior chamber. After enucleation, the patient was treated with chemoradiotherapy combined with autologous stem cell support. However, the patient died due to brain involvement 27 months after enucleation.

\section{Discussion}

In this case, funduscopic examination showed no retinal whitish solitary tumor, which is observed in typical retinoblastoma. Histological examination demonstrated that retinoblastoma cells diffusely invaded the ganglion cell layer without forming masses, assuming ill-defined horizontal growth along the retinal tissue with little vertical growth. Taken together, the diagnosis of this tumor was consistent with diffuse infiltrating retinoblastoma. However, this case was considered unusual because an orange-colored subretinal elevated lesion, a huge choroidal mass, and frequent rosette formations were present, which are not typically observed in patients with diffuse infiltrating retinoblastoma. At histological evaluation, an orange-colored subretinal elevated lesion corresponded to retinoblastoma cell infiltration with rosette formation in the choroid.

Histology of the enucleated eye revealed the presence of retinoblastoma cells in the optic nerve, subarachnoid 
space, choroidal vessels, and trans-scleral canal. The route of brain involvement in this retinoblastoma seems to have been through the optic nerve and subarachnoid space, instead of choroidal vessels or the trans-scleral canal. Therefore, it is consistent with the route of brain involvement in patients with typical retinoblastoma. If the tumor cells had invaded the subarachnoid space, following the choroidal vessels or trans-scleral canal involvement, systemic metastasis would have occurred in the liver and/or lung through blood flow, which was not observed in the present case.

A patient with histologically presumed diffuse infiltrating retinoblastoma was reported, who died due to brain involvement in $1957 .{ }^{3}$ Subsequent reviews disclosed the outcome of consecutive enucleated eyes with diffuse infiltrating retinoblastoma, in which there were no cases of metastases on longterm follow-up. ${ }^{1,2}$ However, the present patient subsequently developed brain involvement, leading to the patient's death 27 months after enucleation. This indicates that a favorable prognosis relies on making a diagnosis much earlier than in this patient. It is known that the presence of a tumor in the subarachnoid space is a risk factor for brain involvement in typical retinoblastoma. ${ }^{4}$ Ophthalmologists should make sure that diffuse infiltrating retinoblastoma has an unfavorable course if its diagnosis is delayed.

\section{Acknowledgment}

This study was supported by the Research Foundation of the Japan Society for the Promotion of Science, and by grants-in-aid from the Scientific Research from The Ministry of Education, Culture, Sports, Science, and Technology.

\section{Disclosure}

The authors report no conflicts of interest in this work.

\section{References}

1. Morgan G. Diffuse infiltrating retinoblastoma. Br J Ophthalmol. 1971;55: 600-606.

2. Shields CL, Ghassemi F, Tuncer S, Thangappan A, Shields JA. Clinical spectrum of diffuse infiltrating retinoblastoma in 34 consecutive eyes. Ophthalmology. 2008;115:2253-2258.

3. Weizenblatt S. Differential diagnostic difficulties in atypical retinoblastoma. Report of a case. Arch Ophthalmol. 1957;58:699-709.

4. Finger PT, Harbour JW, Karcioglu ZA. Risk factors for metastasis in retinoblastoma. Surv Ophthalmol. 2002;47:1-16.
Clinical Ophthalmology

\section{Publish your work in this journal}

Clinical Ophthalmology is an international, peer-reviewed journal covering all subspecialties within ophthalmology. Key topics include: Optometry; Visual science; Pharmacology and drug therapy in eye diseases; Basic Sciences; Primary and Secondary eye care; Patient Safety and Quality of Care Improvements. This journal is indexed on

Submit your manuscript here: http://www.dovepress.com/clinical-ophthalmology-journal

\section{Dovepress}

PubMed Central and CAS, and is the official journal of The Society of Clinical Ophthalmology (SCO). The manuscript management system is completely online and includes a very quick and fair peer-review system, which is all easy to use. Visit http://www.dovepress.com/ testimonials.php to read real quotes from published authors. 